\title{
Pulmonale und pleurale Manifestationen beim Plasmozytom
}

\author{
Pulmonary and Pleural Manifestations of Multiple Myeloma
}

Autoren

Institute
M. Schelle ${ }^{1}$, C. Schreiber ${ }^{1}$, J. Knolle ${ }^{3}$, A. Florschütz ${ }^{2}$, R. Kachel ${ }^{4}$, J. Schreiber ${ }^{1}$

Die Institutsangaben sind am Ende des Beitrags gelistet. eingereicht 1.7.2006

akzeptiert 17.7. 2006

Bibliografie

DOI $10.1055 / \mathrm{s}-2006-944275$

Pneumologie 2006; 60; 743-748

(c) Georg Thieme Verlag KG

Stuttgart · New York

ISSN 0934-8387

\section{Korrespondenzadresse}

PD Dr. med. Jens Schreiber

Städtisches Klinikum Dessau Abteilung für Pneumologie 06847 Dessau jens.schreiber@klinikum-dessau.de

\section{Zusammenfassung}

Das Multiple Myelom ist ein niedrig malignes Non-Hodgkin-Lymphom, das mit einer Infiltration des Knochenmarks durch klonal proliferierende atypische Plasmazellen einhergeht. Die klinische Präsentation wird meist durch die Folgen der Verdrängung der normalen Hämatopoese, der Destruktion von Knochen oder der Immundefizienz bestimmt. Extramedulläre Manifestationen sind vergleichsweise selten. Über pulmonale oder pleurale Beteiligungen wurde kasuistisch berichtet. Bei einem 75-jährigen Patienten wurde ein IgG-sezernierendes Plasmozytom vom Typ Lambda $(\lambda)$ im Stadium III (nach Durie und Salmon) diagnostiziert. Röntgenologisch waren eine diffuse konfluierende noduläre Beherdung beider Lungen und ein ausgedehnter linksseitiger Pleuraerguss nachweisbar. Histologische und immunhistochemische Untersuchungen bestätigten eine diffuse Infiltration sowohl der Lungen als auch der Pleura durch unreife Plasmazellen mit Deposition von IgG- $\lambda$-Leichtketten. Nach einer Vorphasetherapie mit Vincristin und Prednisolon erfolgte eine Polychemotherapie nach dem VAD-Schema, die zu einer Regredienz der pleuropulmonalen Veränderungen führte.

\section{Abstract}

Multiple myeloma is a low malignant, nonHodgkin's lymphoma, which is characterised by infiltration of the bone marrow by clonal prolifaration of atypical plasma cells. Clinical presentation is mostly determined by the sequeles of displacement of normal hemopoiesis, destruction of bones and the immune deficiency. Extramedullary manifestations are relatively rare. Pulmonary and pleural involvement has been described in case reports only. We report on a 75-year-old male patient in whom an IgG-secreting multiple myeloma type lambda $(\lambda)$, stage III (according to Durie and Salmon) has been diagnosed. Chest $\mathrm{X}$-ray and CT revealed a diffuse confluent nodular pattern in the lungs and a large left-sided pleural effusion. Histology and immunohistochemistry confirmed diffuse infiltration of the lungs as well as the pleura by dysmature plasma cells with deposition of $\lambda$-light chains. Preliminary treatment with vincristine and prednisone followed by polychemotherapy according to the VAD scheme was performed, which led to resolution of the pleuropulmonary changes.

\section{Einleitung}

Ein Plasmozytom ist ein niedrig malignes NonHodgkin-Lymphom mit diffuser oder multilokulärer Infiltration des Knochenmarks, das vorwiegend ältere Patienten betrifft. Ein Klon maligner transformierter Plasmazellen bildet Immunglobuline eines einzigen Idiotyps, so genannte monoklonale Immunglobuline (IgG, IgA, IgD, IgE) und/oder Leichtketten (kappa, lambda). Der Hauptmanifestationsort ist somit das Knochenmark und die klinische Präsentation wird durch die Folgen der Verdrängung der normalen Häma- topoese, der Destruktion von Knochen durch eine Osteoklastenaktivierung oder der Immundefizienz bestimmt [1-3]. Extramedulläre Manifestationen sind vergleichsweise selten. Sie können in Form solitärer oder multilokulärer tumorähnlicher Läsionen, den extramedullären Plasmozytomen oder in Form diffuser Infiltrationen von extramedullären Organen durch Plasmazellen und/oder Deposition von monoklonalen Immunglobulinen und/oder leichten Ketten mit konsekutiver Schädigung dieser Organe auftreten [3]. 
Pulmonale oder pleurale Beteiligungen beim Plasmozytom sind Raritäten. Sie können ein breites Spektrum an Manifestationen beinhalten und treten meist in fortgeschrittenen Krankheitsstadien auf. Wir berichten über einen Patienten, bei dem die pulmonale und pleurale Beteiligung eines IgG sezernierenden Plasmozytom vom Typ Lambda die klinische Erstmanifestation darstellte.

\section{Fallbeschreibung}

Ein 75-jähriger Patient wurde wegen zunehmender Dyspnoe bei körperlicher Belastung, eines unproduktiven Hustens, allgemeiner Schwäche und eines Gewichtsverlusts von $10 \mathrm{~kg}$ innerhalb von 6 Wochen stationär eingewiesen.

An Vorerkrankungen bestanden eine koronare Herzkrankheit und eine arterielle Hypertonie. Ein Jahr zuvor war bei einer Routineuntersuchung ein Extragradient in der Gamma-Fraktion der Serumelektrophorese festgestellt und als monoklonales Immunglobulin G-Typ charakterisiert worden. Eine hämatologische Diagnostik führte zur Diagnose einer monoklonalen Gammopathie unbestimmter Signifikanz (MGUS) und zur Initiierung regelmäßiger Verlaufsuntersuchungen.

Bei der klinischen Untersuchung sahen wir einen $188 \mathrm{~cm}$ großen und $83 \mathrm{~kg}$ schweren Patienten in reduziertem Allgemeinzustand. Über den Lungen war ein Vesikuläratmen mit einer Abschwächung des Atemgeräuschs links basal auskultierbar. In diesem Bereich bestand eine Klopfschalldämpfung. Die Leber war $3 \mathrm{~cm}$ unter dem Rippenbogen palpabel und druckschmerzhaft. Der übrige Status praesens war unauffällig.

Bei den laborchemischen Untersuchungen fiel eine starke Erhöhung der Blutsenkungsgeschwindigkeit auf 70/100 mm n.W. auf. Das CrP war mit $5 \mathrm{mg} / \mathrm{l}$ an der oberen Normgrenze (NB: 0 - $5 \mathrm{mg} / \mathrm{l})$. Das Blutbild war mit einem Hämoglobin von 14,5 g/dl (9 mmol/l) (NB: $14-18 \mathrm{~g} / \mathrm{dl})$, Leukozyten von $7,2 \times 10^{3} / \mu \mathrm{l}(\mathrm{NB}$ : $\left.4,3-10 \times 10^{3} / \mu \mathrm{l}\right)$ und Thrombozyten von $170 \times 10^{3} / \mu \mathrm{l}(\mathrm{NB}$ : $\left.140-440 \times 10^{3} / \mu \mathrm{l}\right)$ unauffällig. Das Differenzialblutbild zeigte eine geringe Vermehrung der Lymphozyten auf $46 \%$ und eine leichte Reduktion der segmentkernigen neutrophilen Granulozyten auf 38\%, jedoch keine Blasten oder Plasmazellen. Das Kreatinin im Serum war mit 1,1 mg/l (98 $\mu \mathrm{mol} / \mathrm{l})(\mathrm{NB}: 0,6-1,5 \mathrm{mg} / \mathrm{dl})$ im Normbereich. Es bestanden eine Hyperkalzämie von 5,46 mval/l (2,73 mmol/l) (NB: 2,3 - 5,1 mval/l) und eine Erhöhung der LDH im Serum auf $160 \mathrm{U} / \mathrm{l}(5 \mu \mathrm{mol} / \mathrm{l})$ (NB: 60 - $120 \mathrm{U} / \mathrm{l})$. Das Gesamteiweiß im Serum war mit 72,9 g/l (NB: $66-87 \mathrm{~g} / \mathrm{l}$ ) nicht erhöht. In der Serumelektrophorese (Albumin 48,9\%, $\alpha 1$-Globuline 3,1\%, $\alpha 2$-Globuline 10,6\%, $\beta$-Globuline 9,0\% und $\gamma$-Globuline $28 \%$ ) zeigte sich ein M-Gradient in der Gamma-Fraktion. In der Immunfixation waren im Serum ein monoklonales Paraprotein vom IgG Typ $\lambda$ und im Urin ein Bence-Jones-Protein Typ $\lambda$ nachweisbar ( Abb. 1 ). Immunglobulin G im Serum war auf 26,1 g/l erhöht (Normbereich 7-16 g/l), die Konzentrationen von IgM mit 0,2 g/l (NB: 0,4-2,3 g/l) und IgA mit 0,2 g/l (NB: $0,7-4 \mathrm{~g} / \mathrm{l}$ ) hingegen erniedrigt. In der Durchflusszytometrie war keine pathologische B-Zell-Population im peripheren Blut nachweisbar.

Bei den Röntgen-Untersuchungen der Thoraxorgane zeigte sich über beiden Lungen eine intensive diffuse Beherdung mit unscharf begrenzten und konfluierenden Noduli und ein ausgedehnter linksseitiger Pleuraerguss ( $\mathbf{A b b}$. 2). Die thorakale CT bestätigte beidseitige Pleuraergüsse sowie die diffuse, konfluierende Beherdung des gesamten Lungenparenchyms ( $\mathbf{A b b . 3}$ ).

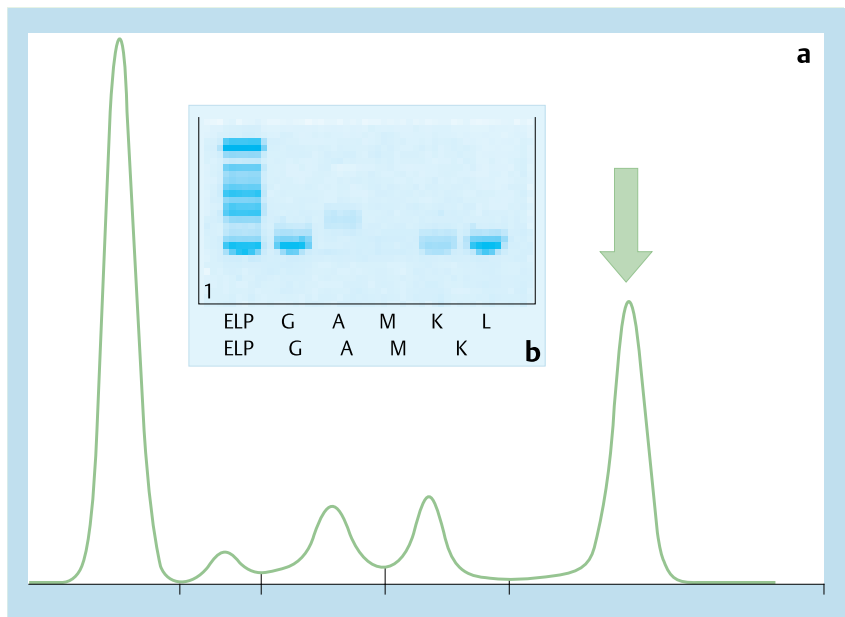

Abb. 1 Serumelektrophorese (a) und Immunelektrophorese (b) mit Nachweis eines Extragradienten durch ein monoklonales IgG Typ Lambda (ELP - Elektrophorese, G - Immunglobulin G, A - Immunglobulin A, M Immunglobulin M, K - Kappa-Leichtketten, L - Lambda-Leichtketten).

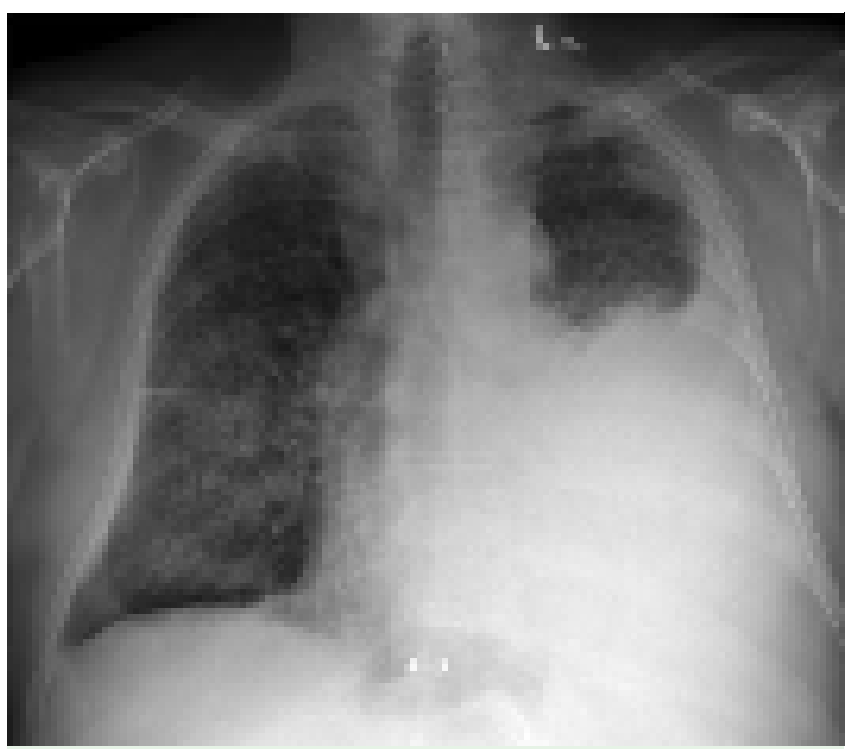

Abb. 2 Röntgenuntersuchung der Thoraxorgane mit Nachweis einer diffusen Beherdung mit unscharf begrenzten und konfluierenden Noduli und eines ausgedehnten linksseitigen Pleuraergusses.

Die Röntgen-Untersuchungen des Beckens, der gesamten Wirbelsäule, des Schädels und der langen Röhrenknochen zeigten multiple Osteolysen im Schädel und in beiden Oberarmen.

Lungenfunktionell waren eine schwergradige restriktive Ventilationsstörung und Einschränkung der Diffusionskapazität nachweisbar. Die Blutgasanalyse in Ruhe wies eine geringe manifeste respiratorische Partialinsuffizienz bei ausgeglichenem Säure-Basen-Status nach.

Das EKG zeigte einen normofrequenten Sinusrhythmus und einen Indifferenztyp ohne Erregungsausbreitungs- und -rückbildungsstörungen.

Echokardiographisch war die linksventrikuläre Pumpfunktion mit einer Ejektionsfraktion von 50\% ohne regionale Dyskinesien global reduziert. Es bestand keine Rechtsherzbelastung.

Die Pleuraergussdiagnostik erbrachte ein Exsudat mit einem Eiweißgehalt von 62,3 g/l und einer LDH von $330 \mathrm{U} / \mathrm{l}$ (10,3 mmol/ 


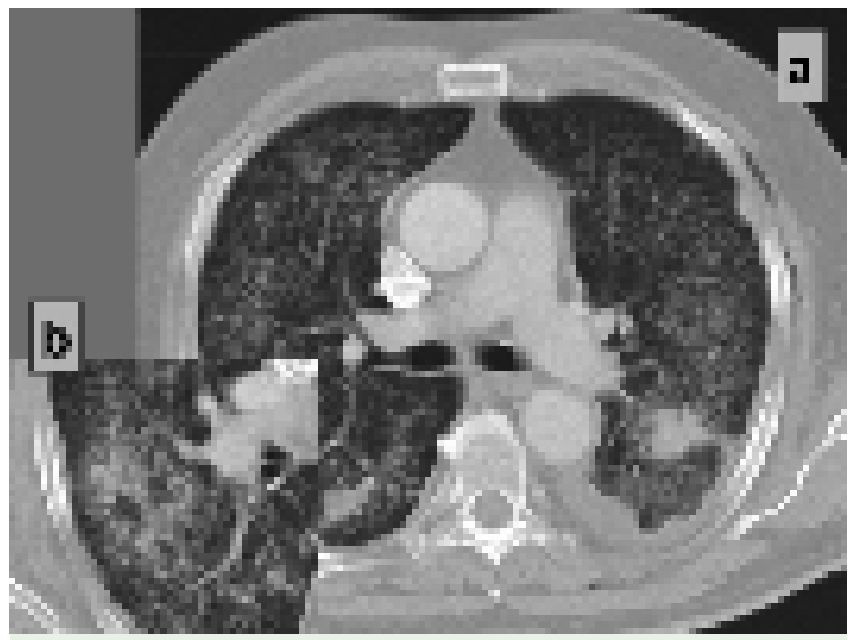

Abb. 3 CT der Thoraxorgane (a: Übersicht, b: Ausschnitt) mit Nachweis einer diffusen Beherdung mit unscharf begrenzten und konfluierenden Noduli. Zusätzlich bestehen links ein minimaler Resterguss und ein Weichteilemphysem nach der Thorakoskopie.

1.s). Die mikrobiologischen Untersuchungen waren negativ, die zytologische Untersuchungen wies neben Leukozyten tumorverdächtige Zellen nach, die auf Grundlage der Immunzytochemie und der in-situ-Hybridisierung als $\lambda$-Leichtketten produzierende Plasmazellen charakterisiert werden konnten ( Abb.4).

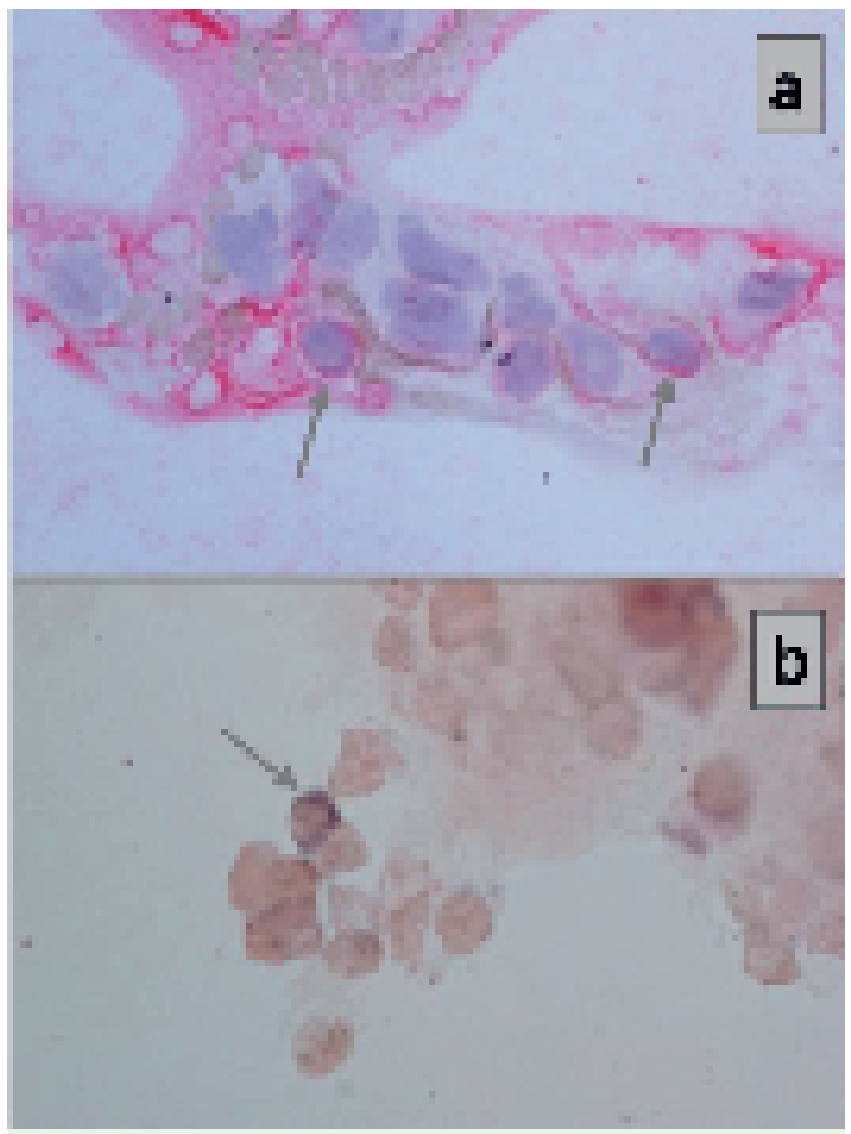

Abb. 4 Zytologische Untersuchungen des Pleuraergusses mit Nachweis von $\lambda$-Leichtketten produzierenden Plasmazellen (a: Immunzytologie $600 \times$, b: In-situ-Hybridisierung $600 \times$ ).
Eine thorakoskopische Inspektion der linken Pleurahöhle zeigte eine wellige, knotige Oberfläche der Lunge. Die Pleura parietalis war diffus hyperämisch mit vereinzelten bizarren polypoiden Auflagerungen ( $\bullet$ Abb. 5). Die histologischen und immunhistochemischen Untersuchungen von Biopsien aus diesen Arealen bewiesen eine diffuse Infiltration durch ein IgG-sezernierendes Plasmozytom vom $\lambda$-Leichtkettentyp ( Abb. 6).

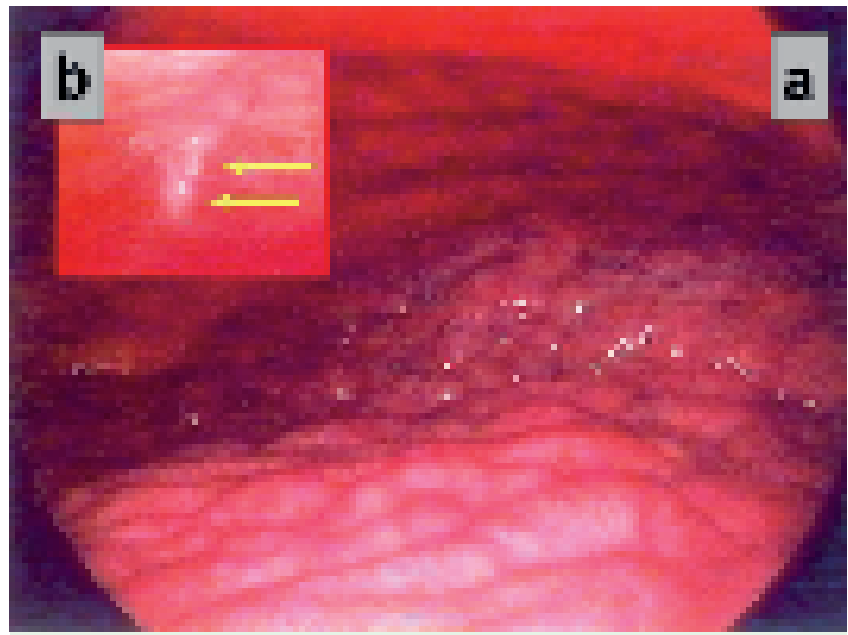

Abb. 5 Thorakoskopischer Situs des linken Hemithorax mit Nachweis einer welligen, knotigen Oberfläche der Lunge und einer hyperämischen Pleura parietalis (a), auf der sich multiple kleine polypoide Auflagerungen zeigten (b).

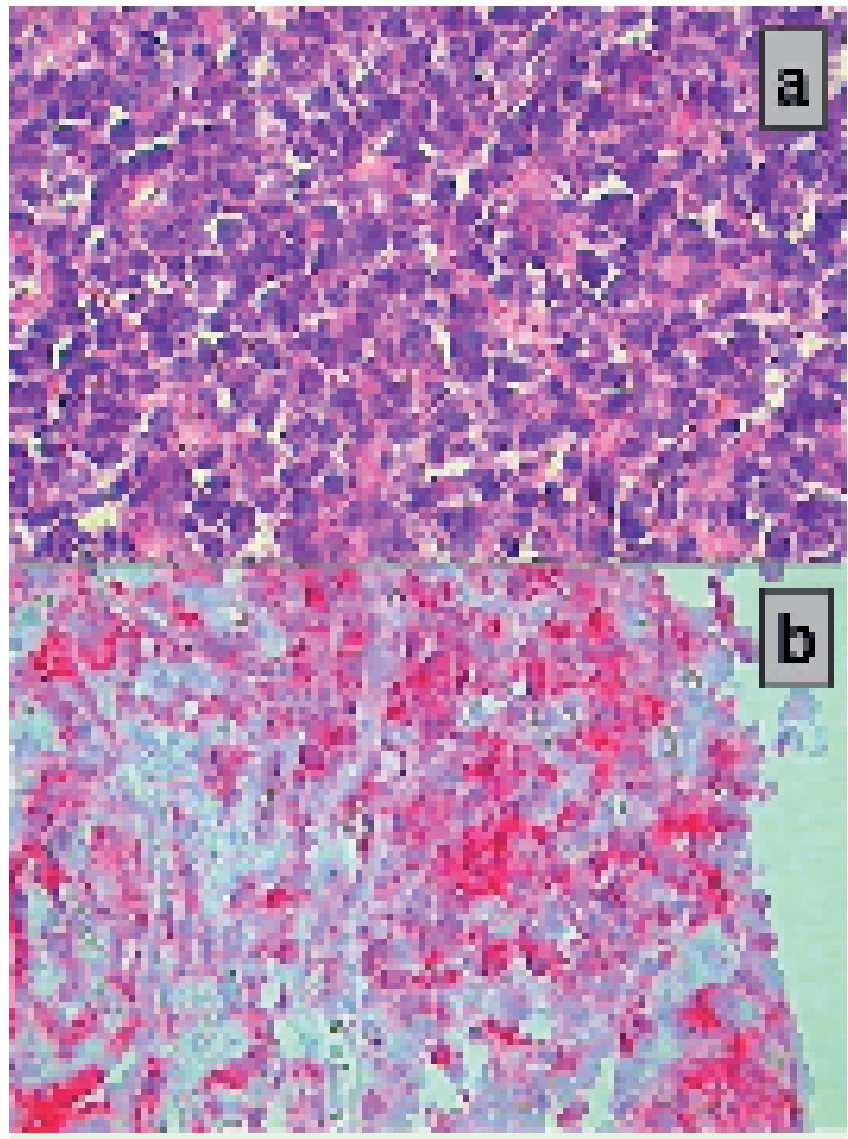

Abb. 6 Histologie von Biopsien aus der Pleura parietalis mit Nachweis einer diffusen Infiltration durch $\lambda$-Leichtketten produzierende Plasmazellen (a: HE-Färbung $400 \times$, b: Immunhistologie $\lambda$-Leichtketten $400 \times$ ). 


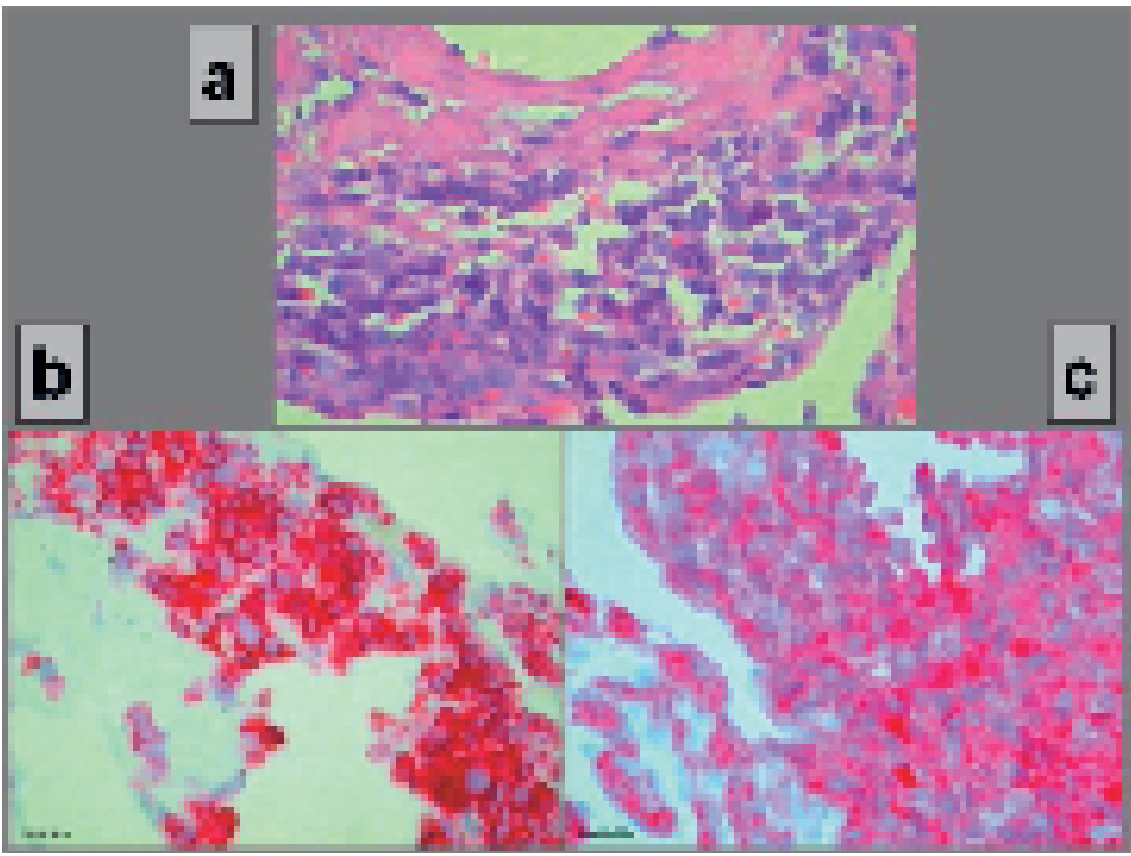

Abb. 7 Histologie transbronchialer Lungenbiopsien mit Nachweis einer diffusen Infiltration durch CD138+, $\lambda$-Leichtketten produzierende Plasmazellen (a: HE-Färbung $400 \times$, b: Immunhistologie CD138 $400 \times$, c: Immunhistologie $\lambda$-Leichtketten $400 \times)$.

Bei einer Bronchoskopie zeigte das zentrale Bronchialsystem den Aspekt einer geringgradigen chronischen Bronchitis mit einer gering vermehrten schleimigen Sekretion. Die mikrobiologische Untersuchung des Bronchialsekrets erbrachte keinen relevanten Keimnachweis. Die histologische Untersuchung von transbronchialen Lungenbiopsien aus dem linken Ober- und Unterlappen bestätigte die Infiltration des Lungenparenchyms durch das Plasmozytom ( Abb. 7).

Im Knochenmark waren die Plasmazellen auf $80 \%$ der kernhaltigen Zellen stark vermehrt. Immunhistochemische Untersuchungen und die In-situ-Hybridisierung verifizierten die Knochenmarkinfiltration durch das IgG-Plasmozytom vom $\lambda$-Leichtkettentyp ( Abb. 8).

Diagnose: Bei dem Patienten lag ein IgG-sezernierendes Plasmozytom vom Typ $\lambda$ im Stadium III (nach Durie und Salmon) ( Tab. 1) mit pleuraler und pulmonaler Beteiligung vor.

Therapie und Verlauf: Auf Grund des reduzierten Allgemeinzustands bei Verdacht auf ein generalisiertes malignes Lymphom sowie des diffusen Organbefalls erfolgte initial eine Vorphasetherapie mit $2 \mathrm{mg}$ Vincristin und $100 \mathrm{mg}$ Prednisolon über 5 Tage. Nach klinischer Stabilisierung und Diagnosesicherung wurde

Tab. 1 Klassifikation des Plasmozytoms nach Durie und Salmon (nach [26])

\begin{tabular}{|c|c|c|}
\hline Stadium I & Stadium II & Stadium III \\
\hline $\begin{array}{l}\text { normale Knochen- } \\
\text { struktur } \\
\text { oder solitäre } \\
\text { Osteolyse }\end{array}$ & $\begin{array}{l}\text { weder Stadium I } \\
\text { noch Stadium III }\end{array}$ & $\begin{array}{l}\text { fortgeschrittene } \\
\text { Knochenläsionen }\end{array}$ \\
\hline $\mathrm{lgG}<50 \mathrm{~g} / \mathrm{l} \mathrm{bzw}$. & $\operatorname{lgG} 50-70 \mathrm{~g} / \mathrm{l} \mathrm{bzw}$ & $\lg G>70 \mathrm{~g} / \mathrm{l} b z w$. \\
\hline $\lg A<30 \mathrm{~g} / \mathrm{l}$ & $\lg A 30-50 \mathrm{~g} / \mathrm{l}$ & $\lg A>50 \mathrm{~g} / \mathrm{l}$ \\
\hline $\begin{array}{l}\text { Leichtkettenaus- } \\
\text { scheidung im Urin } \\
<4 \mathrm{~g} / 24 \mathrm{~h}\end{array}$ & $\begin{array}{l}\text { Leichtkettenaus- } \\
\text { scheidung im Urin } \\
4 \mathrm{~g} / 24 \mathrm{~h}-12 \mathrm{~g} / 24 \mathrm{~h}\end{array}$ & $\begin{array}{l}\text { Leichtkettenaus- } \\
\text { scheidung im Urin } \\
>12 \mathrm{~g} / 24 \mathrm{~h}\end{array}$ \\
\hline $\mathrm{Hb}>10 \mathrm{~g} / \mathrm{dl}$ & $\mathrm{Hb} 8,5 \mathrm{~g} / \mathrm{dl}-10 \mathrm{~g} / \mathrm{dl}$ & $\mathrm{Hb}<8,5 \mathrm{~g} / \mathrm{dl}$ \\
\hline $\begin{array}{l}\text { Serumkalzium } \\
\leq 2,6 \mathrm{mmol} / \mathrm{l}\end{array}$ & $\begin{array}{l}\text { Serumkalzium } \\
<3 \mathrm{mmol} / \mathrm{l}\end{array}$ & $\begin{array}{l}\text { Serumkalzium } \\
>3 \mathrm{mmol} / \mathrm{l}\end{array}$ \\
\hline \multicolumn{3}{|c|}{$\begin{array}{l}\text { Subklassifikation in: } \\
\text { A: Serumkreatinin }<2 \mathrm{mg} / \mathrm{dl} \\
\text { B: Serumkreatinin } \geq 2 \mathrm{mg} / \mathrm{dl}\end{array}$} \\
\hline
\end{tabular}

eine Polychemotherapie nach dem VAD-Schema (Vincristin, Adriamycin, Dexamethason) durchgeführt, die zu einer deutlichen klinischen Besserung und weitgehenden Normalisierung der pleuropulmonalen Veränderungen führte.

\section{Diskussion}

Typisch für das Plasmozytom ist eine diffuse oder multilokuläre Infiltration des Knochenmarks durch einen Klon maligne transformierter Plasmazellen, die monoklonale Immunglobuline oder isolierte leichte Ketten bilden [1,2]. Dieser Pathomechanismus bedingt, dass die klinische Präsentation durch die Folgen der Verdrängung der normalen Hämatopoese, die Destruktion von Knochen durch eine Osteoklastenaktivierung und eine Immundefizienz bestimmt wird [3]. Extramedulläre Manifestationen entweder in Form tumorös imponierender Plasmozytome oder in Form diffuser Organinfiltrationen sind vergleichsweise selten und treten meist erst später im Krankheitsverlauf auf.

So war auch bei unserem Patienten eine diffuse Infiltration des Knochenmarks mit $\operatorname{IgG}$ Typ $\lambda$ sezernierenden Plasmazellen nachweisbar. Die initiale klinische Präsentation war jedoch durch die Folgen der pulmonalen und pleuralen Manifestation der Erkrankung bestimmt. Der klinischen Erstmanifestation ging über ein Jahr der serologische Nachweis eines monoklonalen IgG vom Typ $\lambda$ voraus, der als monoklonale Gammopathie unbestimmter Signifikanz (MGUS) diagnostiziert wurde. Eine MGUS liegt vor, wenn die Konzentration des monoklonalen Immunglobulins $<30 \mathrm{~g} / \mathrm{l}$ und der Anteil von Plasmazellen im Knochenmark $<10 \%$ betragen und wenn keine Organschädigung vorliegt $[1,4,5]$. Dabei handelt es sich um ein relativ häufiges Phänomen. Die Prävalenz der MGUS zeigt einen Anstieg mit zunehmendem Alter und beträgt in der Gruppe der 70-79-jährigen Männer 5,6\% [4]. Die Rate der Progression einer MGUS zum manifesten Plasmozytom beträgt $1 \%$ pro Jahr [5].

Pulmonale und/oder pleurale Manifestationen eines Plasmozytoms sind selten und treten meist spät im Krankheitsverlauf auf. Allerdings sind pulmonale Infiltrate infolge der krankheitsbedingten und therapieinduzierten Immunsuppression bei die- 


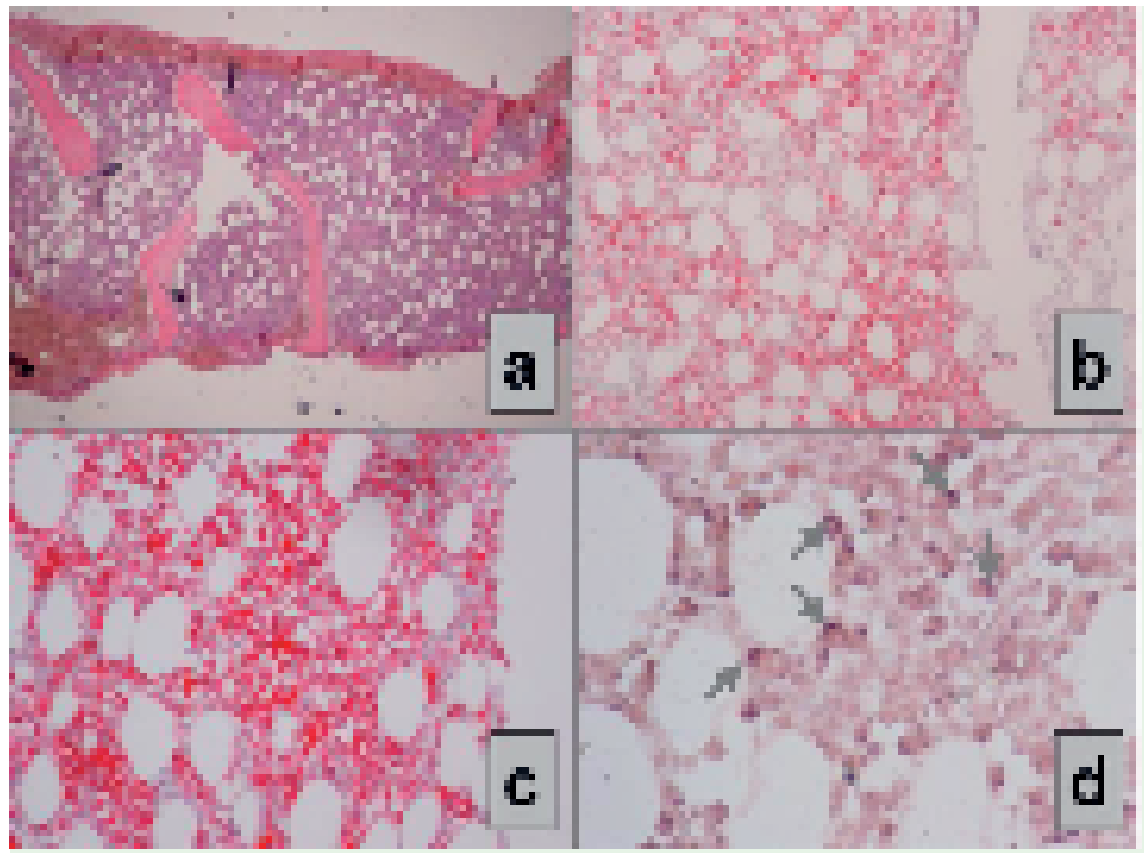

Abb. 8 Knochenmarkhistologie und -zytologie mit Nachweis einer ausgeprägten Infiltration durch CD138+, $\lambda$-Leichtketten produzierende Plasmazellen (> $80 \%$ ) (a: HE-Färbung $4 \times$, b: Immunhistologie CD138 $20 \times$, c: Immunhistologie $\lambda$-Leichtketten $20 \times$, d: In-situ-Hybridisierung $40 \times$ ).

sen Patienten recht häufig und meist infektiöser Genese. In einer Untersuchung an 958 Patienten wurden pulmonale Infiltrate in $10 \%$ nachgewiesen. Lediglich bei 4 Patienten bestand der Verdacht auf eine Infiltration mit Plasmazellen, der letztlich nur bei einem Patienten überzeugend bewiesen wurde. In dieser Studie hatten 85 Patienten (6\%) Pleuraergüsse, die jedoch nur bei 8 Patienten durch eine pleurale Beteiligung der Grundkrankheit bedingt waren [6]. Kasuistisch wurde über 2 Patienten berichtet, bei denen die pleurale Beteiligung an der Grunderkrankung durch eine zytologische Untersuchung verifiziert wurde [7]. In einer weiteren Untersuchung an 38 Patienten mit einem Plasmozytom wurden bei 13 pulmonale Veränderungen nachgewiesen. Davon waren bei zwei Patienten ein solitärer Rundherd, bei zwei weiteren Patienten multiple Rundherde und bei drei Patienten interstitielle Infiltrate nachweisbar. Der Nachweis einer pulmonalen Beteiligung des Plasmozytoms gelang bei $2 \mathrm{~Pa}-$ tienten mit Rundherden und einem Patienten mit einer interstitiellen Lungenerkrankung [8].

Kasuistisch wurde über eine Plasmozytose in der BAL bei einem Patienten mit einem Plasmozytom berichtet [9]. Eine diffuse pulmonale Beteiligung tritt selten im Krankheitsverlauf eines Plasmozytoms auf, mit Ausnahme von aggressiven oder terminalen Phasen der Erkrankung oder bei einer Plasmazellleukämie [10]. Als klinische Erstmanifestation, wie sie auch bei unserem Patienten vorlag, wurde sie von Burkhardt u. Mitarb. beschrieben [11]. In dieser Fallbeschreibung lag gleichzeitig ein Pleuraerguss vor, der jedoch als Folge einer Herzinsuffizienz bei einer plasmozytombedingten myokardialen Schädigung interpretiert wurde [11]. Bei unserem Patienten konnten sowohl eine diffuse pulmonale als auch eine pleurale Infiltration durch das Plasmozytom histologisch verifiziert werden, was nach unseren Recherchen in dieser Form bisher nicht beschrieben wurde.

Eine weitere sehr seltene Manifestation ist das lokalisierte, tumorähnlich imponierende pulmonale, bronchiale oder auch kardiale Plasmozytom [12-14]. Diese können sowohl im Rahmen einer generalisierten Erkrankung als auch sehr selten als primäres Plasmozytom dieser Organe, die dann als solitärer Tumor imponieren, auftreten. Letztere gehen nur selten mit dem Nachweis von monoklonalen Immunglobulinen im Serum einher
[15]. Als primäre Therapie wird eine Resektion empfohlen. Die Prognose eines solitären Plasmozytoms ist günstiger als die des multiplen Myeloms und eine Heilung scheint beim solitären Plasmozytom der Lunge möglich zu sein.

Eine Rarität ist die klinische Präsentation eines Plasmozytoms als diffuse alveoläre Hämorrhagie, die bisher erst zweimal kasuistisch beschrieben wurde. Bei beiden Patienten lag ein IgAPlasmozytom vom Typ Kappa ( $\kappa$ ) vor $[16,17]$, und bei beiden Patienten war die diffuse alveoläre Hämorrhagie nach erfolgreicher Chemotherapie regredient. Bei einem dieser Patienten wurde gleichzeitig eine manifeste pulmonalarterielle Hypertonie nachgewiesen, die auf eine pulmonalvaskuläre und kapilläre Ablagerung von leichten Ketten zurückgeführt wurde [17].

Bei unserem Patienten bestand zusätzlich eine kardiale Schädigung infolge einer koronaren und hypertensiven Herzerkrankung. Eine zusätzliche kardiale Beteiligung des Plasmozytoms, die im Rahmen einer Amyloidose, eines Hyperviskositätssyndroms, einer „light-chain-myocardiopathy“ oder einer high-output Herzinsuffizienz bei ausgedehnten Osteolysen auftreten kann, ließ sich bei unserem Patienten im Rahmen der durchgeführten Untersuchungen nicht nachweisen [3,18-22].

$\mathrm{Zu}$ den bekannten Komplikationen des multiplen Myeloms gehören Ablagerungen von Amyloid mit konsekutiver Schädigung der betroffenen Organe. Eine Amyloidose lag bei unserem Patienten in den untersuchten Biopsien nicht vor, kann in anderen Organen jedoch nicht ausgeschlossen werden. Eine Schädigung von parenchymatösen Organen ist auch durch die Ablagerung von leichten Ketten oder monoklonalen Immunglobulinen ohne Bildung von Amyloid möglich. Typische Beispiele dafür sind die Deposition von leichten Ketten im Nierenparenchym i.S. einer „light chain nephropathy“, die bereits erwähnte Leichtkettenkardiomyopathie und wie bei unserem Patienten die diffuse, klinisch und funktionell relevante Schädigung des Lungenparenchyms [3].

Die wenigen vorliegenden Studien, Fallserien und Kasuistiken zeigen, dass pulmonale Beteiligungen beim Plasmozytom zwar relativ selten, dann aber oft mit einer schlechten Prognose vergesellschaftet sind $[8,10]$. 
Die Standardtherapie des multiplen Myeloms ist eine Chemotherapie in palliativer Zielsetzung $[2,23]$. In den letzten Jahren wurde die Hochdosischemotherapie mit autologer Stammzelltransplantation zu einem Therapieansatz entwickelt, der nach Erreichen einer Remission mit konventioneller Chemotherapie bei geringer therapiebedingter Letalität eine weitere Reduktion der Tumorzellmasse um 90-99\% ermöglicht, was die Prognose der Patienten wesentlich verbessert [24,25].

Zusammenfassend belegt der beschriebene Fall erneut, dass sowohl pulmonale Infiltrate als auch pleurale Manifestationen bei Patienten mit hämatologischen Grunderkrankungen eine breite Differenzialdiagnose umfassen, die die pleuropulmonale Manifestation der Grunderkrankung beinhalten, was beim Plasmozytom ungewöhnlich ist. Die therapierelevante Abgrenzung von Infiltraten und Pleuraergüssen anderer, meist infektiöser Genese erfordert den frühzeitigen Einsatz endoskopisch-bioptischer Methoden.

\section{Institutsangaben}

Städtisches Klinikum Dessau, Akademisches Lehrkrankenhaus der MartinLuther-Universität Halle-Wittenberg, Abteilung für Pneumologie (Ltr.: PD Dr. J. Schreiber), Dessau

2 Städtisches Klinikum Dessau, Akademisches Lehrkrankenhaus der MartinLuther-Universität Halle-Wittenberg, Abteilung für Hämatologie/Onkologie (Ltr.: Dr. A. Florschütz) der Klinik für Innere Medizin (Chefarzt: Prof. Dr. M. Plauth), Dessau

3 Institut für Pathologie (Chefarzt: Dr. J. Knolle), Dessau

${ }^{4}$ Klinik für Diagnostische und Interventionelle Radiologie/Neuroradiologie (Chefarzt: Prof. Dr. R. Kachel), Dessau

\section{Literatur}

1 Goldschmidt H, Cremer FW, Möhler TM. Multiples Myelom Diagnose und Klinik. Onkologe 2004; 10: 809 -819

2 Kyle RA, Rajkumar SV. Multiple myeloma. N Engl J Med 2004; 351: $1860-1873$

3 Malpas JS, Bergsagel DE, Kyle RA et al. Myeloma Biology and Management. 2 ed Oxford, New York, Tokyo: Oxford University Press, 1998

4 Kyle RA, Therneau TM, Rajkumar SV et al. Prevalence of monoclonal gammopathy of undetermined significance. N Engl J Med 2006; 354 : $1362-1369$

5 Kyle RA, Therneau TM, Rajkumar SV et al. A long-term study of prognosis in monoclonal gammopathy of undetermined significance. N Engl J Med 2002; 346: $564-569$

6 Kintzer JS, Rosenow EC, Kyle RA. Thoracic and pulmonary abnormalities in multiple myeloma. A review of 958 cases. Arch Intern Med 1978; 138 : $727-730$
7 Hughes JC, Votaw ML. Pleural effusion in multiple myeloma. Cancer 1979; 44: $1150-1154$

8 Oymak FS, Karaman A, Soyuer I. Pulmonary and chest wall involvement in multiple myeloma. Tuberk Toraks 2003; 51: 27 - 32

9 Menashe P, Stenson W, Reynoso G. Bronchoalveolar lavage plasmocytosis in a patient with plasma cell dyscrasia. Chest 1989; 95: 226-227

10 Garewal H, Durie BGM. Aggressive phase of multiple myeloma with pulmonary plasma cell infiltrates. J Am Med Assoc 1982; 248: 1875 1876

11 Burkhardt O, Dickgreber N, Buhling F et al. Diagnose eines Multiplen Myeloms durch den Nachweis von Plasmazellen in der bronchoalveolären Lavage. Dtsch Med Wochenschr 2003; 128: 1951 - 1954

12 Colosimo $M$, Loi $G$. Extramedullary plasmocytoma with tracheal and pulmonary localization. Nunt Radiol 1967; 33: 1563 - 1571

13 Dornetzhuber $V$, Dobrota $S$. Pathogenesis of pulmonary plasmocytomas (plasmocellular granulomas). Pneumologie 1971; 146: 26 - 33

14 Etienne G, Grenouillet M, Ghiringhelli $C$ et al. Pulmonary plasmacytoma: about two new cases and review of the literature. Rev Med Interne $2004 ; 25$ : $591-595$

15 Koss M, Hochholzer L, Moran C et al. Pulmonary plasmacytomas: a clinicopathologic and immunohistochemical study of five cases. Ann Diagn Pathol 1998; 2: 1 - 11

16 Russi E, Odermatt B, Joller-Jemelka HI et al. Alveolar haemorrhage as a presenting feature of myeloma. Eur Respir J 1993; 6: 267 - 270

17 Schreiber J, Häntze S, Florschütz A et al. Eine seltene Ursache einer diffusen alveolären Hämorrhagie bei einem 45jährigen Mann. Internist, 2006 im Druck

18 Hofer JF, Wimmer G. Severe heart failure from light chain cardiomyopathy (cardiac amyloidosis). Z Kardiol 2003; 92: 90-95

19 Niedeggen A, Breithardt OA, Franke A. Detection of early systolic dysfunction with strain rate imaging in a patient with light chain cardiomyopathy. Z Kardiol 2005; 94: 133-136

20 Schattner A, Epstein M, Berrebi A. Case report: multiple myeloma presenting as a diastolic heart failure with no evidence of amyloidosis. Am J Med Sci 1995; 310: 256-257

21 Kosinski DJ, Roush K, Fraker TD et al. High cardiac output state in patients with multiple myeloma: case report and review of the literature. Clin Cardiol 1994; 17: 678-680

22 McBride W, Jackman JD, Gammon RS et al. High-output cardiac failure in patients with multiple myeloma. N Engl J Med 1988; 319: 1651 1653

23 Ludwig H, Strasser-Weippl K, Zojer N. Konventionelle Therapie des multiplen Myeloms. Onkologe 2004; 10: 820-831

24 Einsele $H$, Straka C. Multiples Myelom - Hochdosistherapie und Stammzelltransplantation. Onkologe 2004; 10: 832 - 842

25 Kroger N, Schwerdtfeger R, Kiehl M et al. Autologous stem cell transplantation followed by a dose-reduced allograft induces high complete remission rate in multiple myeloma. Blood 2002; 100: 755-760

26 Durie BG, Salmon SE. A clinical staging system for multiple myeloma. Correlation of measured myeloma cell mass with presenting clinical features, response to treatment, and survival. Cancer 1975; 36: 842 854 